\title{
PROPRIEDADES VOLUMÉTRICAS E ACÚSTICAS DE SOLUÇÕES LÍQUIDAS BINÁRIAS DE (ADIPATO DE DIETILA + ÁLCOOIS) A DIFERENTES TEMPERATURAS E À PRESSÃO ATMOSFÉRICA
}

\author{
R. A. REIS ${ }^{1}$, R. G. SANTOS ${ }^{1}$, R. B. TÔRRES ${ }^{1}$ \\ ${ }^{1}$ FEI, Departamento de Engenharia Química, \\ E-mail para contato: belchior@fei.edu.br
}

\begin{abstract}
RESUMO - No presente estudo, dados de densidade e de velocidade do som de soluções líquidas binárias de adipato de dietila + metanol, ou + etanol, ou + 1propanol, ou + 1-butanol foram determinadas, em toda faixa de composição, a diferentes temperaturas e á pressão atmosférica. As medidas de densidade e velocidade do som foram determinadas usando um analisador de densidade e velocidade do som fabricado pela Anton Paar (Modelo DSA5000). Os resultados experimentais foram utilizados para calcular o volume molar excesso e o desvio da compressibilidade isentrópica dos sistemas. $\mathrm{O}$ volume molar excesso foi negativo para o sistema contendo metanol e mostrou um comportamento sigmoidal para o sistema contendo etanol. Para os outros sistemas, os valores foram positivos. Os valores do volume molar excesso aumentaram com o aumento da temperatura para todos os sistemas. Os valores do desvio da compressibilidade foram negativos e diminuíram com o aumento da temperatura para todos os sistemas estudados.
\end{abstract}

\section{INTRODUÇÃO}

As funções excesso representam um caminho qualitativo e quantitativo para descrever o quanto uma solução real desvia da idealidade. Essas funções são usadas em uma grande variedade de campos da ciência, incluindo química e engenharia química. Em especial, o volume excesso é uma grandeza de grande interesse para estudo, pois a complexidade associada ao seu comportamento juntamente com a facilidade de obtê-lo experimentalmente e com grande precisão fazem com que essa grandeza seja de grande importância no desenvolvimento e teste de modelos e teorias de soluções.

A importância do conhecimento do desvio da compressibilidade isentrópica como uma função da composição e temperatura consiste no fato da velocidade do som ser uma propriedade importante para a descrição de fenômenos acústicos e para o desenvolvimento de equações de estado (Span, 2000). Várias propriedades termofísicas podem ser derivadas de dados exatos de velocidade do som (Trusler, 1991).

$\mathrm{O}$ adipato de dietila, denominado hexanodioato de dietila, é um éster que pode ser obtido a partir de ácido adípico, através de uma reação de carboxilação. Por possuir uma massa molecular 
de aproximadamente $202.25 \mathrm{~g} / \mathrm{mol}$ e apresenta baixa volatilidade à temperatura ambiente. Estudos apontam sua possível utilização como aditivo ao diesel, como demonstrado por Ren et al. (2007). A adição desse componente ao diesel provocou um aumento de duração do tempo da ignição e da quantidade de calor liberada na combustão.

O presente estudo teve como objetivo a determinação experimental de medidas de densidade e velocidade do som de soluções líquidas binárias de adipato de dietila, + metanol, ou + etanol, ou + 1-propanol, ou + 1-butanol, em toda faixa de composição, na faixa de temperatura entre $293,15 \mathrm{~K}$ e $308,15 \mathrm{~K}$ e à pressão atmosférica. Os resultados experimentais foram usados para calcular o volume molar excesso, $\left(V_{\mathrm{m}}^{\mathrm{E}}\right)$, e o desvio da compressibilidade isentrópica, $(\Delta \kappa)$.

\section{PARTE EXPERIMENTAL}

Todos os reagentes utilizados no estudo apresentaram os seguintes valores de pureza, conforme especificações encontradas nos rótulos dos fabricantes: Adipato de dietila (Aldrich, pureza $\geq 99,0 \%$ ), metanol (Merck, pureza $\geq 99,9 \%$ ), etanol (Merck, pureza $\geq 99,5 \%$ ), 1-propanol (Merck, pureza $\geq 99,8 \%$ ) e 1-butanol (Merck, pureza $\geq 99,5 \%$ ). Os reagentes não passaram por processo de purificação adicional. A Tabela 1 apresenta uma comparação entre os valores de densidade encontrados na literatura e os resultados experimentais.

Tabela 1: Comparação entre as densidades experimentais e aquelas encontradas na literatura dos componentes puros utilizados nesse trabalho.

\begin{tabular}{lll}
\hline Componente & Densidade, $\rho^{293,15} / \mathrm{g} \cdot \mathrm{cm}^{-3}$ & Experimental, $\rho^{293,15} / \mathrm{g} \cdot \mathrm{cm}^{-3}$ \\
\hline Adipato de dietila & 1,0066 (Comuñas et al., 2008) & 1,006771 \\
Metanol & 0,7915 (Pereira et al., 2003) & 0,791351 \\
Etanol & 0,789454 (Kijevcanin et al., 2007) & 0,789775 \\
1-Propanol & 0,80350 (Zarei et al., 2008) & 0,804713 \\
1-Butanol & 0,80956 (Liau et al., 1998) & 0,812548 \\
\hline
\end{tabular}

O volume molar excesso e o desvio da compressibilidade isentrópica foram determinados indiretamente através de medidas de densidade e da velocidade do som dos componentes puros e das soluções. A densidade e a velocidade do som foram determinadas usando um analisador de densidade e velocidade do som fabricado pela Anton Paar (Modelo DAS 5000). A resolução das medida da densidade e da velocidade do som foi, respectivamente, de $1 \times 10^{-6} \mathrm{~g} \cdot \mathrm{cm}^{-3} \mathrm{e} 0,1 \mathrm{~m} \cdot \mathrm{s}^{-1}$.

As soluções foram preparadas em frascos de $10 \mathrm{~cm}^{3}$ e levadas imediatamente aos equipamentos para que fossem feitas as leituras da densidade e da velocidade do som. As amostras foram injetadas nos equipamentos usando seringas especiais. As composições das soluções foram determinadas através da determinação das massas dos componentes puros, usando uma balança analítica fabricada pela OHAUS (Modelo Adventurer, resolução de 1x 10 g). 


\section{RESULTADOS}

$\mathrm{O} V_{\mathrm{m}}^{\mathrm{E}}$ foi calculado a partir da densidade dos componentes puros e das respectivas soluções utilizando a seguinte equação:

$$
V_{\mathrm{m}}^{\mathrm{E}}=x_{1} M_{1}\left(\frac{1}{\rho}-\frac{1}{\rho_{1}}\right)+x_{2} M_{2}\left(\frac{1}{\rho}-\frac{1}{\rho_{2}}\right) \text {, }
$$

onde $x_{i}, M_{i}, \rho_{i}$ representam a fração molar, a massa molar e a densidade do componente $i$ e $\rho$ é a densidade da solução.

O desvio da compressibilidade isentrópica foi calculado pela equação:

$$
\Delta \kappa=\kappa-\left(x_{1} \kappa_{1}+x_{2} \kappa_{2}\right)
$$

com

$\kappa=\frac{1}{\rho u^{2}}$,

onde $\kappa$ é a compressibilidade isentrópica, $u$ é a velocidade do som e $\rho$ a densidade.

Os valores experimentais das grandezas determinadas foram ajustados através de um polinômio do tipo Redlich-Kister (Redlich e Kister, 1948). Várias versões desses polinômios são encontradas na literatura para descrever o comportamento de funções excesso. No presente estudo foi tulizada a seguinte equação:

$$
Y_{\mathrm{m}}^{\mathrm{E}}=x_{1}\left(1-x_{1}\right) \sum_{j=0}^{j=n} A_{j}\left(1-2 x_{2}\right)^{j},
$$

onde $Y$ é $\boldsymbol{V}_{\mathrm{m}}^{\mathrm{E}}$ ou $\Delta \kappa$.

\section{DISCUSSÕES}

As Figuras 1 e 2 apresentam os valores de volume molar excesso e do desvio da compressibilidade isentrópica para os sistemas e temperaturas estudados. 

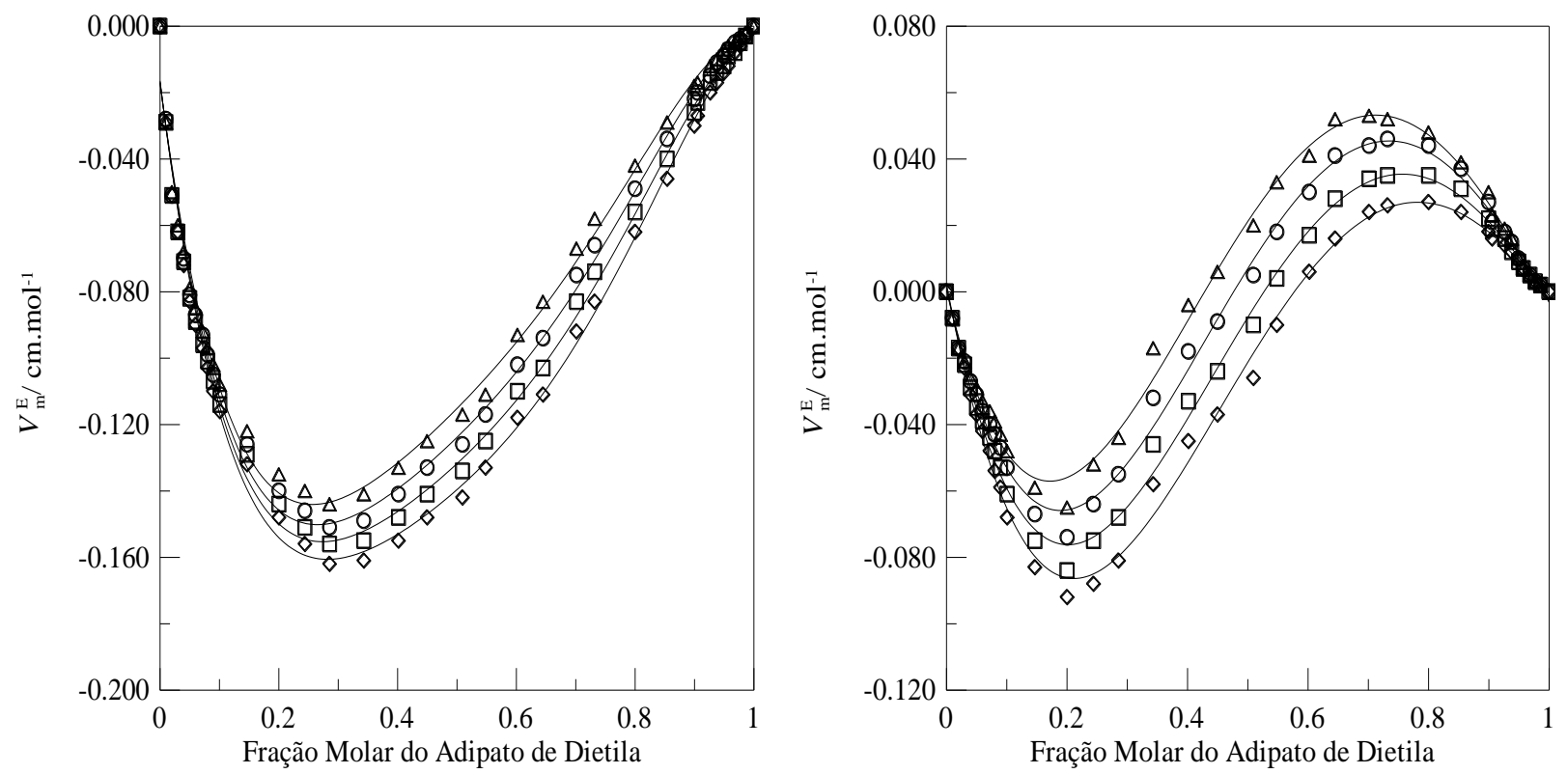

A

B
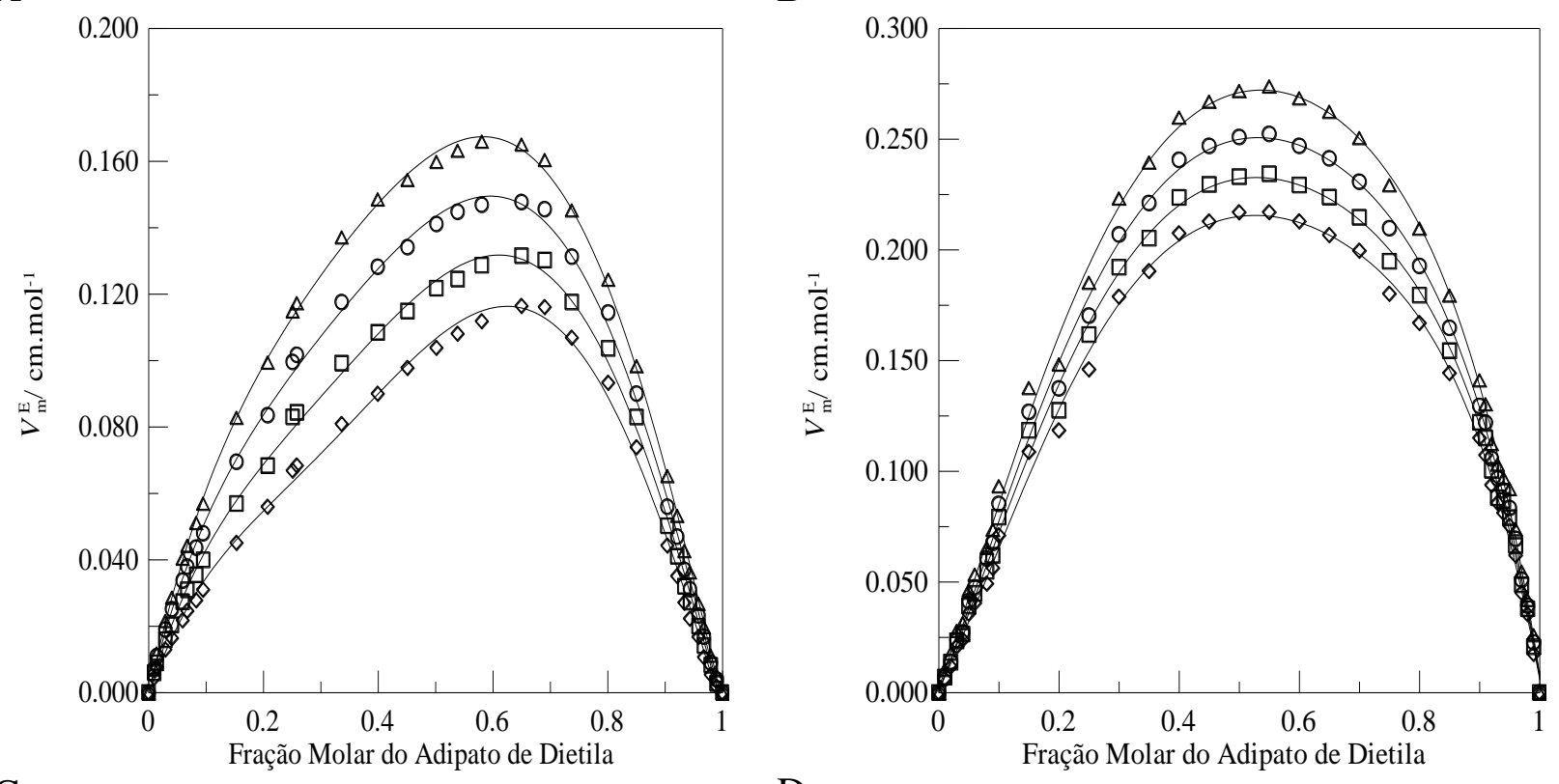

$\mathrm{D}$

Figura 1 - Valores do volume molar excesso, em função da fração molar do adipato de dietila, para o sistema adipato de dietila + álcool a diferentes temperaturas: $\diamond, 293,15 \mathrm{~K} ; \square, 298,15 \mathrm{~K}$; O, $298.15 \mathrm{~K} ; \Delta, 303,15$ K. — Redlich-Kister. A, metanol; B, etanol; C, 1-propanol; D, 1butanol. 


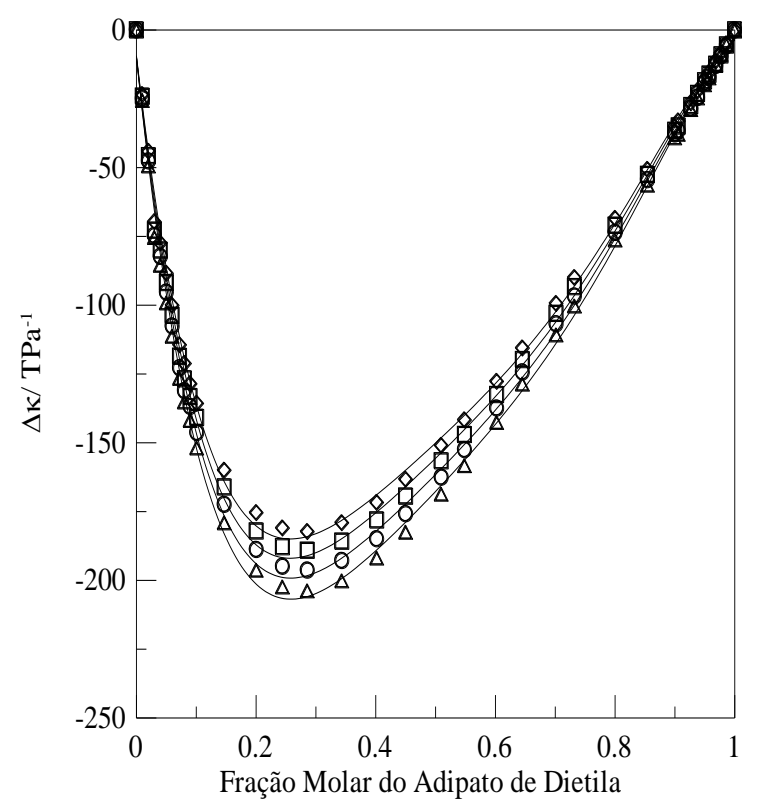

A

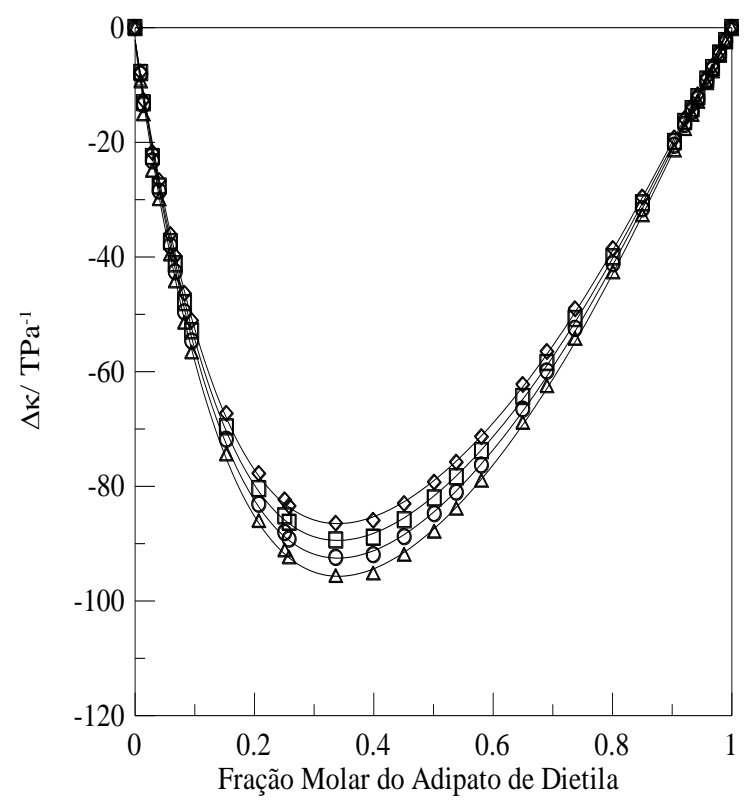

$\mathrm{C}$

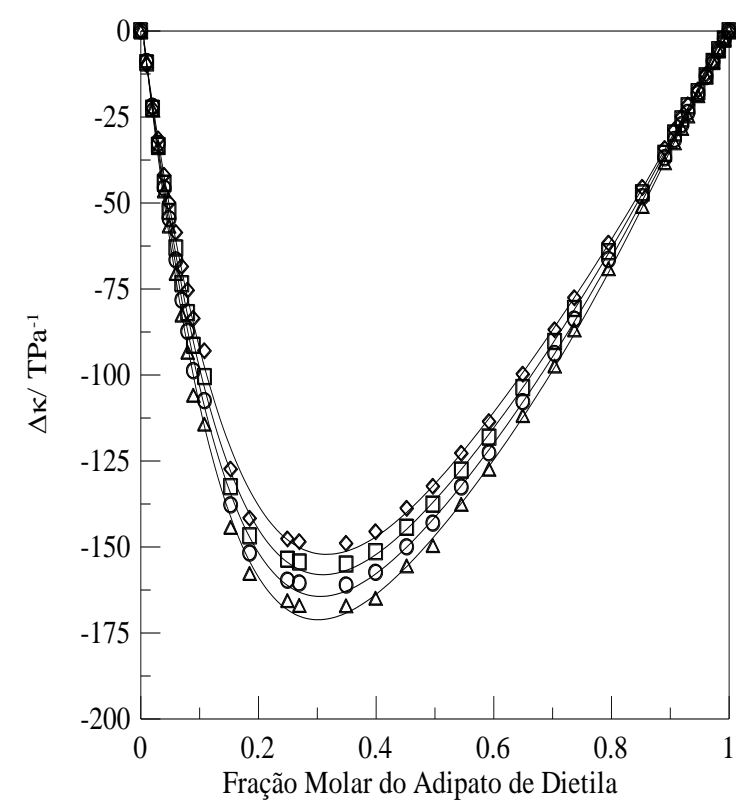

B

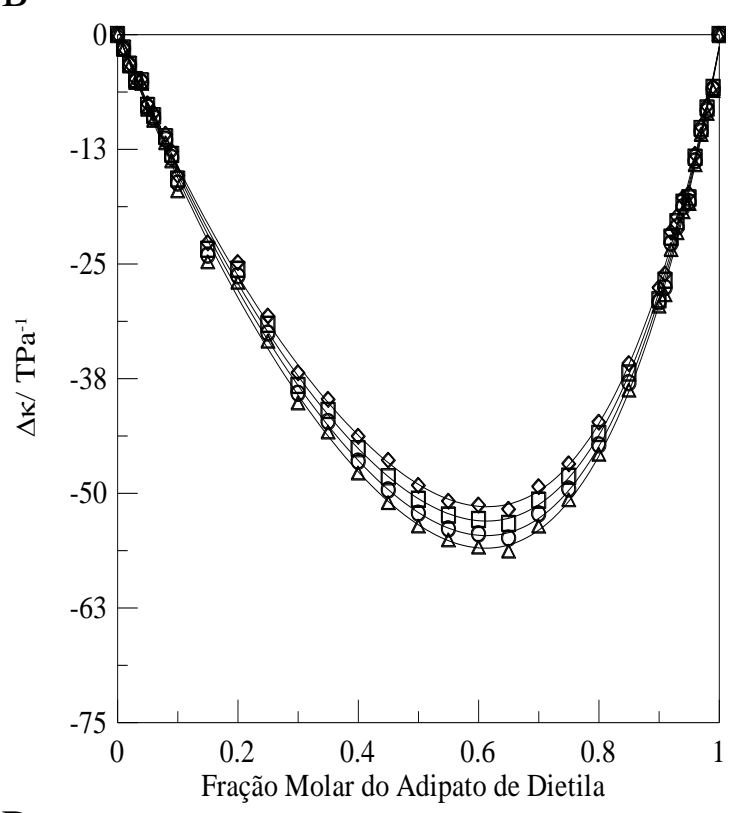

Figura 2 - Valores do desvio da compressibilidade isentrópica, em função da fração molar do adipato de dietila, para o sistema adipato de dietila + álcool a diferentes temperaturas: $\diamond, 293,15$ K; $\square, 298,15$ K; O, 298.15 K; $\Delta, 303,15$ K. —— Redlich-Kister. A, metanol; B, etanol; C, 1propanol; D, 1-butanol. 


\section{9 a 22 de outubro de 2014 \\ Florianópolis/SC}

Os resultados experimentais do volume molar excesso apresentaram valores negativos para o sistema contendo metanol, e um comportamento sigmoidal para o sistema contendo etanol. Para os sistemas contendo 1-propanol e 1-butanol, os valores do $V_{\mathrm{m}}^{\mathrm{E}}$ foram positivos. Para todos os sistemas, os valores $V_{\mathrm{m}}^{\mathrm{E}}$ aumentaram, isto é, tornaram-se menos negativos com o aumento da temperatura. Já os valores de $\Delta \kappa$ foram negativos para todos os sistemas em toda faixa de composição, e tornaram-se mais negativo com o aumento da temperatura.

A magnitude e o sinal do $V_{\mathrm{m}}^{\mathrm{E}}$ são resultados de diferentes efeitos, os quais podem ser divididos em físicos, químicos e estruturais: (1) as interações físicas envolvendo, principalmente, interações não específicas, contribuem para valores positivos de $V_{\mathrm{m}}^{\mathrm{E}}$; (2) a quebra da ordem da estrutura líquida na mistura também contribui positivamente no valor de $V_{\mathrm{m}}^{\mathrm{E}}$; (3) interações químicas ou específicas, tais como formação de complexos e ligações de hidrogênio entre as moléculas dos constituintes presentes na solução contribuem para valores negativos de $V_{\mathrm{m}}^{\mathrm{E}} ;$ (4) efeitos estruturais advindo das acomodações intersticiais devido à diferença no volume molar e no volume livre entre as moléculas dos componentes presentes na solução também contribuem negativamente no valor de $V_{\mathrm{m}}^{\mathrm{E}}$. De acordo com os resultados experimentais, possivelmente os efeitos (3) e (4) devem predominar sobre os efeitos (1) e (2) para os sistemas contendo metanol e etanol. Para os outros dois sistemas os efeitos (1) e (2) devem ser preponderantes.

As moléculas do adipato e dos alcoóis são polares e apresentam grupos funcionais que podem realizar ligações de hidrogênio. Assim, é possível que ligações de hidrogênio entre esses grupos funcionais também estejam ocorrendo e provocando uma redução no volume molar da solução formada. Outro indício de que isso possa estar ocorrendo é o comportamento do volume molar excesso com a temperatura. Efeitos químicos diminuem com o aumento da temperatura. $\mathrm{O}$ aumento da temperatura pode estar diminuindo as ligações de hidrogênio entre o adipato e o metanol o que leva ao aumento do volume molar excesso com o aumento da temperatura. Para o sistema contendo etanol, o aumento da concentração do adipato na mistura pode estar quebrando a estrutura do álcool levando a uma expansão do volume. Como consequência os valores do volume molar excesso mudam de sinal e torna-se positivo a partir da fração molar de $x 1=0,4$.

Os valores negativos do desvio da compressibilidade isentrópica significam que a solução é menos compressível do que os componentes puros e que o aumento da temperatura potencializa esse efeito. Conforme a solução reduz sua compressibilidade, aumenta a mobilidade das moléculas presentes no meio, o que facilita a propagação da velocidade do som. Sendo a compressibilidade isentrópica inversamente proporcional ao quadrado da velocidade do som, quanto maior for a velocidade do som no meio, menor será a compressibilidade.

\section{CONCLUSÕES}

No presente estudo, dados experimentais de densidade e velocidade do som para sistemas líquidos binários de (adipato de dietila + alcoóis) têm sido usados para calcular o volume molar 


\section{9 a 22 de outubro de 2014 \\ Florianópolis/SC}

excesso e o desvio da compressibilidade dos sistemas. Para os sistemas adipato de dietila + metanol, ou + etanol parecem que os efeitos (3) e (4) foram mais importantes para descrever o comportamento do volume molar excesso. Contudo, para o sistema adipato de dietila + etanol, o volume molar excesso foi negativo na região de baixa concentração, o que deve prevalecer nessa região os efeitos (3) e (4). À medida que a concentração do adipato de dietila aumenta, o volume molar excesso muda de sinal, passando a assumir valores positivos e, portanto, prevalecendo possivelmente os efeitos (1) e (2). Para o sistema contendo 1-propanol e 1- butanol, os valores $V_{\mathrm{m}}^{\mathrm{E}}$ são todos positivos. Para todos os sistemas estudados os valores do volume molar excesso aumentaram com o aumento da temperatura. Os valores do desvio da compressibilidade isentrópica foram negativos, em toda faixa de composição, para todos os sistemas estudados e tornaram-se mais negativos com o aumento da temperatura. Isso significa que a solução é menos compressível do que os componentes puros e que o aumento da temperatura potencializa esse efeito.

\section{AGRADECIMENTOS}

Os autores agradecem a FEI e a FAPESP pelo auxilio financeiro através do Projeto 09/14556-5.

\section{REFERÊNCIAS}

AL-JIMAZ, A.S.; AL-KANDARY, J.A.; ABDUL-LATIF, A.M. Densities and viscosities for binary mixtures of phenetole with 1-pentanol, 1-hexanol, 1-heptanol, 1-octanol, 1nonanol, and 1-decanol at different temperatures. Fluid Phase Equilibria, v. 218, n. 2, p. 247-260, 2004.

COMUÑAS, M.J.P.; BAZILE, J.; ANTOINE BAYLAUCQ, A.; BONED, C. Density of diethyl adipate using a new vibrating tube densimeter from (293.15 to 403.15$) \mathrm{K}$ and up to 140 MPa. Calibration and Measurements. J. Chem. Eng. Data, v. 53, p. 986-994, 2008.

EGOROV, G. I.; MAKAROV, D. M. Volumetric properties of binary mixtures of glycerol + tert-butanol over the temperature range 293.15 to $348.15 \mathrm{k}$ at atmospheric pressure. $J$ Solution Chem., v. 41, p. 536-554, 2012.

GIRO, F.; GONÇALVES, M.F.; FERREIRA, A.G.M.; FONSECA, I.M.A. Viscosity and density data of the system water $+n$-pentyl acetate + methanol: Calculations with a modified Redlich-Kwong-Soave equation of state. Fluid Phase Equilibr., v. 204, n. 2, p. 217-232, 2004.

GRUNBERG, L; NISSAN, AH. Mixture law for viscosity. Nature, v. 164, n. 4175, p. 799800, 1949.

HERIC, E.L.; BREWER, J.G. Viscosity of some binary liquid nonelectrolyte mixtures. $J$. Chem. Eng. Data, v. 12, p. 574-583, 1967.

HIND, R.K.; MCLAUGHLIN, E.; UBBELOHIDE, A.R. Structure and viscosity of liquids camphor + pyrene mixtures. Trans. of the Faraday Soc., . v. 56, n. 3, p. 328-330, 1960. 
KAPADI, U.R.; HUNDIWALE, D.G.; PATIL, N.B.; LANDE, M.K.; PATIL, P.R. Studies of viscosity and excess molar volume of binary mixtures of propane-1,2 diol with water at various temperatures. Fluid Phase Equilibr., v. 192, n. 1-2, p. 63-70, 2001.

KATTI, P.K.; CHAUDHRI, M.M. Viscosities of binary mixtures of benzyl acetate with dioxane, aniline, and m-cresol. J. Chem. Eng. Data, v. 9, p. 4432-443, 1964.

KIJEVČANIN, M. L.; ŠERBANOVIĆ, S. P.; RADOVIĆ, I. R.; DJORDJEVIĆ, B. D.; TASIĆ, A. Ž. Volumetric properties of the ternary system ethanol + chloroform + benzene at temperature range (288.15-313.15) K: Experimental data, correlation and prediction by cubic EOS. Fluid Phase Equilib., v. 251, p. 78-92, 2007.

OSWAL, S.L.; DESAI, H.S. Studies of viscosity and excess molar volume of binary mixtures: 1. Propylamine +1 -alkanol mixtures at 303.15 and 313.15 K. Fluid Phase Equilibr., v. 149, n. 1-2, p. 359-376, 1998.

REDLICH, O.; KISTER, T. Algebraic representation of thermodynamic properties and the classification of solutions. Ind. Eng. Chemistry, v. 40, p. 345-348, 1948.

REN, Y.; HUANG, Z.; MIAO, H.; JIANG, D.; ZENG, K.; LIU, B.; WANG, X. Combustion and emission characteristics of a direct-injection diesel engine fueled with diesel-diethyl adipate blends. Energy Fuels, v. 21, n. 3, p. 1474-1482, 2007.

RODRIGUEZ, A.; CANOSA, J.; DOMINGUEZ, A.; TOJO, J. Viscosities of dimethyl carbonate with alcohols at several temperatures: UNIFAC-VISCO interaction parameters (-OCOO-/alcohol). Fluid Phase Equilibr., v. 216, n. 1, p. 167-174, 2004.

ZAREI, H. A.; ASADI, S.; ILOUKHANI, H. Temperature dependence of the volumetric properties of binary mixtures of (1-propanol, 2-propanol and 1,2-propanediol) at ambient pressure (81.5 kPa). J. Molecular Liq., v. 141, p. 25-30, 2008. 